\title{
Transcriptional Analysis of Acinetobacter sp. neg1 Capable of Degrading Ochratoxin A
}

\author{
Vania C. Liuzzi ${ }^{1+}$, Francesca Fanelli ${ }^{1+}$, Mariana Tristezza ${ }^{2}$, Miriam Haidukowski ${ }^{1}$, \\ Ernesto Picardi ${ }^{3}$, Caterina Manzari ${ }^{4}$, Claudia Lionetti ${ }^{3}$, Francesco Grieco ${ }^{2}$, \\ Antonio F. Logrieco', Michael R. Thon ${ }^{5}$, Graziano Pesole ${ }^{3,4,6}$ and Giuseppina Mulè ${ }^{1 *}$ \\ ' Institute of Sciences of Food Production, National Research Council, Bari, Italy, ${ }^{2}$ Institute of Sciences of Food Production, \\ National Research Council, Lecce, Italy, ${ }^{3}$ Department of Biosciences, Biotechnologies and Biopharmaceutics, University of \\ Bari, Bari, Italy, ${ }^{4}$ Institute of Biomembranes and Bioenergetics, National Research Council, Bari, Italy, ${ }^{5}$ Center Hispano-Luso \\ de Investigaciones Agrarias, University of Salamanca, Salamanca, Spain, ${ }^{6}$ Consorzio Interuniversitario Biotecnologie, Trieste, \\ Italy
}

OPEN ACCESS

Edited by:

Weiwen Zhang,

Tianjin University, China

Reviewed by:

Raffaele A. Calogero,

University of Turin, Italy

Lucilla lacumin,

University of Udine, Italy

*Correspondence:

Giuseppina Mulè

giuseppina.mule@ispa.cnr.it

${ }^{\dagger}$ These authors have contributed equally to this work.

Specialty section:

This article was submitted to Microbiotechnology, Ecotoxicology and Bioremediation,

a section of the journal

Frontiers in Microbiology

Received: 08 July 2016 Accepted: 22 December 2016 Published: 09 January 2017

Citation:

Liuzzi VC, Fanelli F, Tristezza $M$,

Haidukowski M, Picardi E,

Manzari C, Lionetti C, Grieco F, Logrieco AF, Thon MR, Pesole G and Mulè G (2017) Transcriptional Analysis of Acinetobacter sp. neg 1 Capable of Degrading Ochratoxin A.

Front. Microbiol. 7:2162.

doi: 10.3389/fmicb.2016.02162
Ochratoxin A (OTA) is a nephrotoxic and potentially carcinogenic mycotoxin produced by several species of Aspergillus and Penicillium, contaminating grapes, wine and a variety of food products. We recently isolated from OTA contaminated soil vineyard a novel free-living strain of Acinetobacter sp. neg1, ITEM 17016, able to degrade OTA into the non-toxic catabolic product ochratoxin $\alpha$. Biochemical studies suggested that the degradation reaction proceeds via peptide bond hydrolysis with phenylalanine (Phe) release. In order to identify genes responsible for OTA degradation we performed a differential gene expression analysis of ITEM 17016 grown in the presence or absence of the toxin. Among the differentially expressed genes, six peptidases up-regulated at $6 \mathrm{~h}$ were identified. The degrading activity of the carboxypeptidase PJ_1540 was confirmed in vitro in a heterologous system. The enrichment analysis for Gene Ontology terms confirmed that OTA degradation proceeds through peptidase activities and revealed the over-representation of pathways related to Phe catabolism. These results indicate that Phe may represent an energy source for this Acinetobacter sp. neg1 strain and that OTA degrading reaction triggers the modulation of further catabolic activities.

Keywords: peptidase, phenylalanine, mycotoxin, pathway analysis, food safety

\section{INTRODUCTION}

Ochratoxin A (OTA) is a mycotoxin produced by several species of Aspergillus and Penicillium, which contaminates the food and feed chain world-wide. Aspergillus carbonarius, A. ochraceus, considered the main OTA producers (Pitt, 1987; Abarca et al., 1994; Bayman et al., 2002; Frisvad et al., 2006). Cereals and cereal products (Araguás et al., 2005; Makun et al., 2007; Duarte et al., 2010), coffee, grapes, wine (Battilani et al., 2004), dried vine fruits (Covarelli et al., 2012), coffee beans (Taniwaki et al., 2003), spices (Romani et al., 2000), but also livestock products, such as milk (Breitholtz-Emanuelsson et al., 1993) and meat (Perši et al., 2014) are the main sources of human OTA exposure (Miraglia and Brera, 2002). A. alliaceus, A. steynii, A. westerdijkiae, A. niger, Penicillium nordicum and $P$. verrucosum are 
With regards to the chemical structure, OTA is a phenylalanine-dihydroisocoumarine derivative, composed of a 7-carboxy5-chloro-8-hydroxy-3,4-dihydro-3-R-methylisocoumarin (ochratoxin $\alpha$ - OT $\alpha$ moiety) moiety and a L- $\beta$-phenylalanine molecule (Phe), which are linked through the 7-carboxy group by an amide bond.

OTA is primarily known for its nephrotoxic effects. It has been extensively demonstrated that it is the most causal determinant of porcine nephropathy, as well as of human endemic Balkan nephropathy and chronic interstitial nephropathy in North Africa (Pfohl-Leszkowicz and Manderville, 2007; Stefanović and Polenaković, 2009). It is classified by the International Agency for Research on Cancer (IARC) as possibly carcinogenic to humans (group 2B; IARC, 1993). Furthermore, in 1998 the Scientific Committee on Food (SCF) concluded that OTA has carcinogenic, nephrotoxic, teratogenic, immunotoxic and possibly neurotoxic properties (SCF, 1998).

Due to the toxin stability to both temperature and hydrolysis, the current strategies to control OTA levels are mainly preventive, based on pre-harvest management, and aimed to avoid fungal colonization.

Post-harvest strategies are classified into physical, chemical or biological (Varga et al., 2010). Although the first two are generally reported as effective in reducing OTA levels, they induced relevant changes in sensory and nutritional qualities of food. Moreover, toxic residues in the final products may pose safety concern.

For these reasons, biological methods have been increasingly considered as an alternative to physical and chemical treatments. Numerous bacteria, protozoa, and fungi are able to degrade OTA. Also purified enzymes, including lipases from Aspergillus niger, carboxypeptidase A and some commercial proteases have been reported to perform this reaction (Abrunhosa et al., 2010). OTA degradation may occur via two kinds of pathways: the hydrolysis of or the lactone ring or the amine bond, by peptidase activity, that links the Phe to the OT $\alpha$ moiety.

In the first case, the final degradation product is an opened lactone form of OTA, which is of similar toxicity to OTA. The second mechanism can be considered a real detoxification pathway because leads to two non-toxic catabolic products: Phe and OT $\alpha$. The essential non-toxicity of OT $\alpha$ was demonstrated both in prokaryotic and eukaryotic cellular systems as well as in animal models (Xiao et al., 1996).

We recently isolated from vineyard soils a novel freeliving strain of Acinetobacter sp. neg1, ITEM 17016, capable of degrading OTA into the non-toxic catabolic product OT $\alpha$. ITEM 17016 genome was sequenced and the phylogenomic analysis placed A. sp. neg1 as sister to Acinetobacter gyllenbergii (Fanelli et al., 2015).

Numerous peptidase-encoding genes are present in the genome of ITEM 17016. In order to develop biotechnological applications of the enzymatic degrading activities we described the identification of potential candidate genes and pathways involved in OTA degradation through comparative transcriptional analysis. Moreover, we cloned the identified selected peptidase in a heterologous system and tested the detoxification capability of the recombinant protein.

\section{MATERIALS AND METHODS}

\section{Strain}

The Acinetobacter sp. neg1 strain used in this study was ITEM 17016 from Agri-Food Toxigenic Fungi Culture Collection of the Institute of Sciences of Food Production, CNR, Bari ${ }^{1}$. It was isolated from a soil sample in a Negroamaro vineyard in Cellino S. Marco (BR, Italy), reported as contaminated by high levels of OTA in previous years (Somma et al., 2012). ITEM 17016 was demonstrated to be capable of degrading OTA in vitro (De Bellis et al., 2015; Fanelli et al., 2015).

\section{Growth Curve and OTA Degradation Assay}

Bacteria were precultured overnight. A bacterial suspension was inoculated in minimal medium peptone (MMP) medium [2.5 g/l $\mathrm{K}_{2} \mathrm{HPO}_{4}$ (Baker, Deventer, Holland), $1.0 \mathrm{~g} / \mathrm{l}\left(\mathrm{NH}_{4}\right)_{2} \mathrm{HPO}_{4}$ (SIGMA, Steinheim, Germany), $0.2 \mathrm{~g} / \mathrm{l} \mathrm{MgSO}_{4} * 7 \mathrm{H}_{2} \mathrm{O}$ (Carlo Erba, Rodano (MI), Italy), 0.5\% Bacto Peptone (Biolife, Milano, Italy)] and cultured at $28^{\circ} \mathrm{C}$ with shaking at $120 \mathrm{rpm}$ up to Optical Density (OD) of 0.5. A spectrophotometer Ultrospec 3100 pro (Amersham pharmacia biotech) was used for the $\mathrm{OD}_{600}$ measurement. For the degradation assay, bacteria were inoculated at $2 \%$ in a final volume of $5 \mathrm{ml}$ of MMP medium supplemented with OTA $(1 \mu \mathrm{g} / \mathrm{ml})$ and grown for $144 \mathrm{~h}$. Bacterial growth and cell viability were monitored by measuring the $\mathrm{OD}_{600}$ and plate counting for each time point $(0,3,6,12,24$, $48,72,96,144 \mathrm{~h})$. For the plate counting, serial dilutions of the bacterial suspensions were prepared in $0.85 \% \mathrm{NaCl}$ (AppliChem, Darmstatd, Germany), 0.1\% Tween (Fisher Scientific, Bridgewater, NJ, USA) $\mathrm{H}_{2} \mathrm{O}$ sterile solution and spread-plated on pre-poured PCA plates. Plates were incubated at $28^{\circ} \mathrm{C}$ overnight and $\mathrm{CFU}$ were counted. Each experiment was performed in triplicate. One milliliter of the culture was centrifuged and the supernatant was used for the chemical analysis.

\section{RNA Extraction and Library Construction}

At 6 and $12 \mathrm{~h}, 1 \mathrm{~mL}$ from each triplicate of cultures grown in the presence or absence of OTA was collected by centrifugation at $13,000 \mathrm{rpm}$ for $10 \mathrm{~min}$. Pellets were then resuspended in RNAlater RNA Stabilization Reagent (Qiagen, Hilden, Germany), centrifuged at $13,000 \mathrm{rpm}$ for $10 \mathrm{~min}$ and stored at $-80^{\circ}$ until extraction.

Total RNA was extracted using the RNeasy Mini Kit (Qiagen, Hilden, Germany), according to the manufacturer's instructions. The quality and the quantity of the RNA sample were analyzed and verified using a NanoDrop1000 spectrophotometer (Thermo Fisher Scientific, Rodano (MI), Italy) and 2100 Bioanalyzer (Agilent Technologies, Santa Clara, CA, USA), obtaining RIN (RNA Integrity Number) values ranging from 7 to 9.

Total RNA from each preparation (about $1 \mu \mathrm{g}$ ) was depleted of ribosomal RNA using the Ribo-Zero Magnetic kit for Gram negative bacteria (Part \#15065382, November 2014, Illumina, San Diego, CA, USA) and purified with RNeasyMinElute

\footnotetext{
${ }^{1}$ www.ispa.cnr.it/Collection
} 
Cleanup kit (QIAGEN, Hilden, Germania) according to the supplied instructions. The cDNA sequencing libraries for whole transcriptome analysis were prepared using SureSelect StrandSpecific RNA Library Prep for Illumina Multiplexed Sequencing (Version C.0, December 2014, Agilent Technologies, Santa Clara, CA, USA). Briefly, RNA was chemically fragmented prior to reverse transcription reaction. The first-strand cDNAs were synthesized with random hexamer primers and followed by second-strand cDNA synthesis and ends repair. Then, the cDNAs were $3^{\prime}$-adenylated, ligated to the adaptors, amplified, and indexed. The libraries quality was checked by using the Agilent DNA 1000 assay 2100 (Agilent Technologies, Santa Clara, CA, USA) and quantified by using the Nano-Drop 3300 Fluorospectrometer (Thermo-Scientific, Waltham, MA, USA) with the Quant-IT PicoGreen assay kit (Life-Technologies, Carlsbad, CA, USA). All the indexed library produced were subjected to paired-end $2 \times 120$ bp sequencing on the Illumina MiSeq platform, generating for each sample approximately $3 \mathrm{M}$ of $120 \mathrm{bp}$ paired-end reads.

\section{Data Analysis}

The quality of raw data was initially checked by FastQC software ${ }^{2}$. Then RNAseq reads were trimmed using Trim Galore ${ }^{3}$ in order to remove low quality regions and adaptors. The cleaned reads were mapped against the reference genome JSZD01 of Acinetobacter sp. neg1 (Fanelli et al., 2015) using GSNAP (Wu and Nacu, 2010). Finally, CuffDiff software, implemented in the CyVerse (iPlant) Discovery Environment, was used to calculate DE genes between the two conditions (Trapnell et al., 2010). Gene Ontology enrichment analysis was performed using the Java-based tool Bingo (Maere et al., 2005), included as a plugin in Cytoscape (Shannon et al., 2003). The hypergeometric test and a FDRadjusted $\mathrm{p}$-value threshold of 0.05 were used to select significantly enriched pathways.

\section{Cloning and Expression of Peptidase-Coding Genes}

Preparation of genomic DNA from ITEM 17016 was performed according to the method reported by Cappello et al. (2008). Polymerase chain reaction (PCR) was performed with primers designed to amplify the DNA segments encoding the upregulated genes in the presence of OTA, flanked by NcoI and XhoI restriction sites, using the Phusion ${ }^{\circledR}$ High-Fidelity DNA Polymerase (New England Biolabs, Ipswich, MA, USA; Table 1). PCR was performed according to the following steps: denaturation for $30 \mathrm{~s}$ at $98^{\circ} \mathrm{C} ; 35$ cycles: $10 \mathrm{~s}$ at $98^{\circ} \mathrm{C}, 30 \mathrm{~s}$ at $56^{\circ} \mathrm{C}, 30 \mathrm{~s}$ at $72^{\circ} \mathrm{C}$; final extension for $10 \mathrm{~min}$ at $72^{\circ} \mathrm{C}$. The obtained amplicon was purified by using the PureLink PCR Purification Kit (Thermo Fisher Scientific, Waltham, MA, USA) and ligated into the cloning vector pCR-II-BluntTOPO (Thermo Fisher Scientific, Waltham, MA, USA). E. coli competent cells One Shot TOP10 (Thermo Fisher Scientific, Waltham, MA, USA) were transformed with the construct, following supplier's instructions. Plasmid DNA was extracted

${ }^{2}$ http://www.bioinformatics.babraham.ac.uk/projects/fastqc/

${ }^{3}$ http://www.bioinformatics.babraham.ac.uk/projects/trim_galore/
TABLE 1 | Nucleotide sequences of primers used in this study with Ncol (forward primers) and $\mathrm{Xhol}$ (reverse primers) restriction sites in italics.

\begin{tabular}{llc}
\hline Primer & Sequence & Tm \\
\hline PJ15_1540_forward & 5'-AAACCATGGTTTATCCT & $56^{\circ} \mathrm{C}$ \\
& AAAATGCTAGGC-3' & \\
PJ15_1540_reverse & 5'-AAACTCGAGGAACAAGTTGC & \\
& TAAAGAACATTTTG-3' & \\
\hline
\end{tabular}

using the PureLink Quick Plasmid Miniprep Kit (Thermo Fisher Scientific, Waltham, MA, USA) and the recombinant clones were subjected to sequence analysis using the ABI PRISM 3130 Genetic Analyzer (Applied Biosystems, Foster City, CA, USA). Plasmid DNA was digested with NcoI and XhoI (Thermo Scientific, USA) and the insert was ligated into the expression vector pET-28a(+; Novagen, Madison, WI, USA) using T4 DNA Ligase (Thermo Fisher Scientific, Waltham, MA, USA) following supplier's instructions. The construct was used to transform E. coli competent cells BL21(DE3) and BL21-CodonPlus(DE3)RIL (Agilent Technologies, Santa Clara, CA, USA).

Cells carrying the recombinant plasmid were inoculated in $3 \mathrm{~mL}$ of Luria-Bertani (LB; Sigma-Aldrich S.R.L., Milan, Italy) broth supplemented with $50 \mu \mathrm{g} / \mathrm{mL}$ kanamycin (Sigma-Aldrich S.R.L., Milan, Italy), $34 \mu \mathrm{g} / \mathrm{mL}$ chloramphenicol (Sigma-Aldrich S.R.L., Milan, Italy) and cultured overnight at $37^{\circ} \mathrm{C}$ with rotary shaking (250 rpm). One $\mathrm{ml}$ aliquot of cell culture was transferred into $9 \mathrm{~mL}$ of fresh LB supplemented with antibiotics and cultured $2 \mathrm{~h}$ at $37^{\circ} \mathrm{C}$ with rotary shaking (250 rpm). Isopropyl- $\beta-\mathrm{D}-1-$ tiogalattopiranoside (IPTG; Sigma-Aldrich S.R.L., Milan, Italy) was added to induce the expression of the recombinant protein. Different IPTG concentrations $(0.1,0.5,1.0$, and $2.0 \mathrm{mM})$, incubation time $(2,4$, and $6 \mathrm{~h}$ ) and temperature (37 and $30^{\circ} \mathrm{C}$ ) were tested. After the induction, cells were harvested by centrifugation at $4,000 \mathrm{~g}$ for $5 \mathrm{~min}$ at $4^{\circ} \mathrm{C}$. Bacterial pellets and supernatants were kept at $-80^{\circ} \mathrm{C}$ until further analysis. Total proteins from supernatant liquid were precipitated with trichloroacetic acid/acetone (Sambrook et al., 1989) and then analyzed by SDS-PAGE as previously described (Gallo et al., 2012).

\section{OTA Degradation by Recombinant Peptidases}

The degrading activity of the recombinant peptidases was tested as follows: after IPTG induction, bacterial pellets were suspended in prechilled TS Buffer (Tris 0.25 M, NaCl 1.37 M, KCl 0.027 M, Sigma-Aldrich S.R.L., Milan, Italy) and lysed in a French press (thrice at $1700 \mathrm{psi}$ ). Cell lysate and supernatant liquid were incubated overnight with $1 \mu \mathrm{g} / \mathrm{mL}$ of OTA at $28^{\circ} \mathrm{C}$ and the percentage of OTA degradation and OT $\alpha$ production were measured by high-performance liquid chromatography with fluorescence detection (HPLC-FLD) analysis (see section HPLCFLD Analysis). Each experiment was performed in triplicate.

\section{Chemical Analyses Chemicals and Reagents}

Acetonitrile $(\mathrm{ACN})$, methanol $(\mathrm{MetOH})$ and acetic acid were purchased from Mallinckrodt Baker (Milan, Italy). Ultrapure 
water was produced by a Millipore Milli-Q system (Millipore, Bedford, MA, USA). Ammonium acetate (for mass spectrometry) was from Sigma-Aldrich (Milan, Italy). Micro Spin Filter Tubes $(0.20 \mu \mathrm{m}$, regenerated cellulose) were purchased from Phenomenex (Phenomenex, Torrance, CA, USA).

\section{Preparation of Standards}

Ochratoxin A stock solution was prepared by dissolving the solid commercial toxin (Sigma-Aldrich, USA) in MetOH $(1 \mathrm{mg} / \mathrm{ml})$. Appropriate aliquots of the stock solution were brought to dryness and reconstituted with $\mathrm{ACN}$-water-acetic acid (99:99:2 $\mathrm{v} / \mathrm{v} / \mathrm{v})$ to obtain standard solutions of OTA within the range $0.05-0.10 \mu \mathrm{g} / \mathrm{ml}$. The standard of OT $\alpha$ was purchased from Biopure (Romer Labs Diagnostic GmbH, Austria) at a concentration of $10 \mu \mathrm{g} / \mathrm{ml}$ in ACN. Aliquots of the stock solution were brought to dryness and reconstituted with $\mathrm{ACN}$-wateracetic acid $(99: 99: 2, \mathrm{v} / \mathrm{v} / \mathrm{v})$ to obtain standard solutions of OT $\alpha$ from 0.01 to $0.10 \mu \mathrm{g} / \mathrm{ml}$.

\section{HPLC-FLD Analysis}

Decimal dilutions of the supernatants [both from ITEM 17016 and from transformed BL21-CodonPlus(DE3)-RIL cells] were prepared with ACN-water-glacial acetic acid (99:99:2, v/v/v). Samples were filtered using RC $0.2 \mu \mathrm{m}$ micro spin filter tubes. Fifty microliters were injected into the HPLC apparatus (Technology series 1260, Agilent, Germany) with a full loop injection system. Direct injection of liquid culture was possible since no interfering peaks eluted at retention times of OT $\alpha$ and OTA. The fluorimetric detector was set at wavelengths of $340 \mathrm{~nm}$ (excitation) and $460 \mathrm{~nm}$ (emission). The analytical column was a Symmetry C18 ( $5 \mu \mathrm{m}, 150 \mathrm{~mm} \times 4.6 \mathrm{~mm}$; Waters, USA) with a guard column inlet filter $(0.5 \mu \mathrm{m} \times 3 \mathrm{~mm}$ diameter; Rheodyne Inc., USA). The initial composition of the mobile phase was $55 \%$ solvent A (water-acetic acid, 99:1, v/v) and $45 \%$ solvent B (ACN-acetic acid, 99:1, v/v) and was kept constant for $14 \mathrm{~min}$, then solvent B was linearly increased to $80 \%$ in $2 \mathrm{~min}$ and kept constant for $4 \mathrm{~min}$. The mobile phase flow rate was $1 \mathrm{ml} / \mathrm{min}$. OT $\alpha$ and OTA were measured by comparing peak areas with calibration curves; the retention times were 9.69 and $11.25 \mathrm{~min}$, respectively. The detection limit was $0.1 \mathrm{ng} / \mathrm{ml}$ for OTA and OT $\alpha$ (based on a signal-noise ratio 3:1).

\section{HPLC-HRMS Analysis}

Structural confirmation of OT $\alpha$ and identification of others possible degradation metabolites were performed by LC-HRMS. Twenty milliliters of supernatants were also analyzed by LCHRMS according to the procedure described by Gallo et al. (2012). The column was a Kinetex C18 column (100 mm by $2.10 \mathrm{~mm}, 2.6 \mu \mathrm{m}$; Phenomenex, Torrance, CA, USA). The mobile phase was a multistep gradient of water (solvent $\mathrm{A}$ ) and MetOH (solvent B), both containing $0.5 \%$ acetic acid and $1 \mathrm{mM}$ ammonium acetate. The initial composition of the mobile phase was $20 \%$ solvent B, after $3 \mathrm{~min}$ the proportion was set at $40 \%$ and then linearly increased to $63 \%$ in $35 \mathrm{~min}$ and kept constant for $11 \mathrm{~min}$. HPLC-HRMS analyses were performed on a benchtop single-stage mass spectrometer (Exactive) ${ }^{\mathrm{TM}}$ equipped with a heated electrospray ion source (HESI II; Thermo Fisher Scientific,

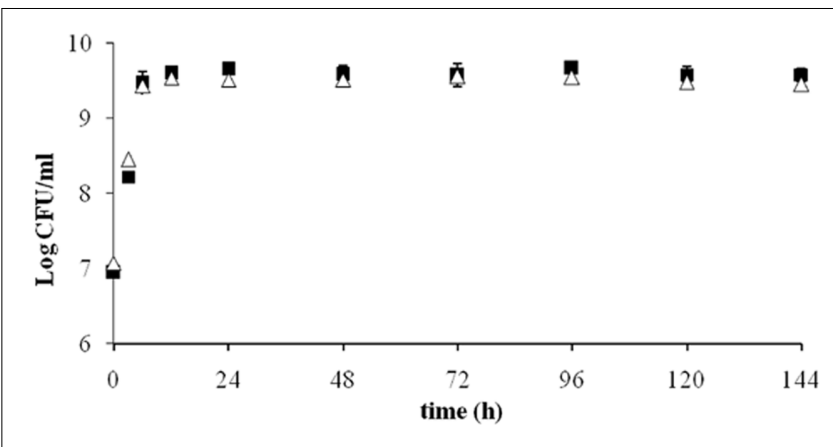

FIGURE 1 | Viable ITEM 17016 cells in minimal medium peptone (MMP) medium (squares) and MMP supplemented with OTA $(1 \mu \mathrm{g} / \mathrm{ml}$; triangle) at $28^{\circ}$.

Bremen, Germany), coupled to an HPLC system Accela (Thermo Fisher Scientific, San Jose, CA, USA). The HESI II interface was used in the positive-ion mode, and the scan range was 50.2 to $1,003.0 \mathrm{~m} / z$, with a resolution power of $100,000 \mathrm{FWHM}$ (full width at half maximum). Other settings were as follows: sheath and auxiliary gas flow rates, 30 and 10 arbitrary units, respectively; sweep gas, 0 arbitrary units; capillary temperature, $300^{\circ} \mathrm{C}$; capillary voltage, $4 \mathrm{kV}$. The Xcalibur software (version 2.1.0, Thermo Fisher Scientific) was used for data acquisition and processing.

\section{RESULTS}

\section{Growth Curve}

The growth curve of ITEM 17016 in MMP medium and MMP supplemented with OTA is shown in Figure 1. The initial inoculum was approximately $7 \mathrm{Log} \mathrm{CFU} / \mathrm{mL}$ and reached the maximum of $9.6 \mathrm{Log} \mathrm{CFU} / \mathrm{mL}$ after a $12 \mathrm{~h}$. Number of viable cells slightly decreased after $96 \mathrm{~h}$. Comparison between the two curves did not show notable differences.

\section{OTA Degradation by ITEM 17016}

The results of OTA degradation by ITEM 17016 are shown in Figure 2. OTA level decreased rapidly in the first $6 \mathrm{~h}$ of incubation. After this period the degrading activity was slightly reduced until $48 \mathrm{~h}$. The maximum rapidity of OTA decrease, after ITEM 17016 reached its highest viable cell count (Figure 1), was measured between $48-72 \mathrm{~h}$ and $96-144 \mathrm{~h}$. At $144 \mathrm{~h}$ more than $70 \%$ of OTA was degraded to OT $\alpha$. OT $\alpha$ content increased steadily in the medium at the same speed by which OTA was degraded.

\section{Transcriptional Profiling and Differential Expression Analysis}

The analysis pipeline of the RNA seq data has been performed as described in Section "Data Analysis." A preliminary evaluation of the raw data quality was performed by FastQC software, indicating that more that $95 \%$ of reads per sample showed an average quality score higher than 30 . Next, TrimGalore 


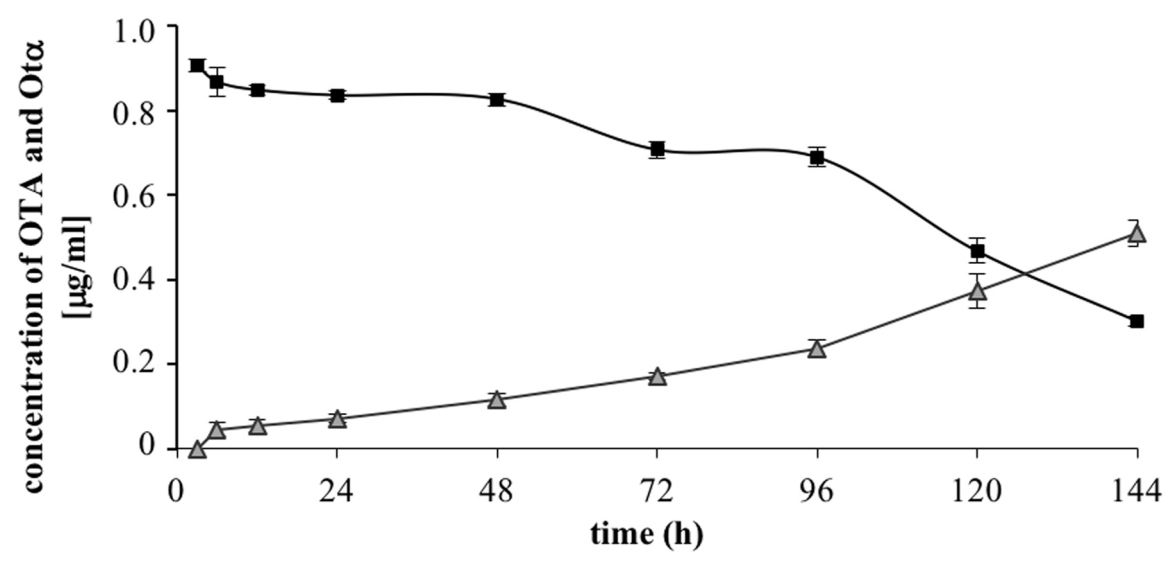

FIGURE 2 | Changes of OTA (squares) and OT $\alpha$ (triangles) in MMP medium at $28^{\circ}$ during the degradation assay.

software was applied to remove adapters and trim low quality regions at $3^{\prime}$ end of reads using a minimum quality score cut-off of 25. GSNAP software was used to map reads to the reference genome JSZD01 of $A$. sp. neg1. The resulting alignment files, in BAM format, were provided to Cuffdiff, which is part of the extensive Cufflinks package developed for the identification of differentially expressed (DE) genes and transcripts (Trapnell et al., 2010). This software estimates the expression changes, controls for variability across replicate libraries and uses the Benjamini-Hochberg procedure to control the False Discovery Rate (FDR). CuffDiff was launched in the Discovery Environment of CyVerse (iPlant Collaborative) interface and platform ${ }^{4}$. The CuffDiff analysis showed 111 and $88 \mathrm{DE}$ genes with a FDR-adjusted $p$-value $<0.05$ between OTA-treated and control samples after 6 and $12 \mathrm{~h}$, respectively (Supplementary Data Sheets 1 and 2). Among them, six genes code for peptidases and resulted up-regulated at $6 \mathrm{~h}$ (Table 2). PJ15_1540 and PJ15_2908 both code for a serine-type D-Ala-DAla carboxypeptidase similar to the ASAG1 carboxypeptidase of Bacillus amyloliquefaciens, which was reported to degrade OTA in vitro (Chang et al., 2015). PJ15_1540 was selected for the experimental validation based on its higher identity (29\%) with the ASAG1 carboxypeptidase.

\section{Experimental Validation}

\section{Expression of Peptidase-Coding Genes in E. coli}

The amplified DNA fragment of the PJ15_1540 gene showed an electrophoretic mobility on agarose gel corresponding to its theoretical size of 1212 bp (not shown). No mutations were found in the amplicon as compared to the deposited gene sequence. The PJ15_1540 purified fragment was ligated in the vector pET-28a(+) and cloned into E. coli BL21(DE3). However, the recombinant plasmid did not accomplish any detectable expression of the protein in this host bacterial strain (not shown). Thus, the construct pET-28a(+)-PJ15_1540 was used to transform E. coli BL21-CodonPlus-RIL, which is able to express the tRNA genes for arginine (AGA and

${ }^{4} \mathrm{http} / / /$ www.iplantcollaborative.org/
TABLE 2 | Genes coding for peptidases resulted up-regulated at $6 \mathrm{~h}$.

\begin{tabular}{|c|c|c|}
\hline Gene name & Gene ontology molecular function & $\begin{array}{c}\text { log2 (fold } \\
\text { change) }\end{array}$ \\
\hline PJ15_1540 & $\begin{array}{l}\text { serine-type D-Ala-D-Ala } \\
\text { carboxypeptidase activity } \\
\text { [GO:0009002] }\end{array}$ & 0.608439 \\
\hline PJ15_2703 & $\begin{array}{l}\text { metalloendopeptidase activity } \\
\text { [GO:0004222] }\end{array}$ & 0.844572 \\
\hline PJ15_2908 & $\begin{array}{l}\text { serine-type D-Ala-D-Ala } \\
\text { carboxypeptidase activity } \\
\text { [GO:0009002] }\end{array}$ & 1.02051 \\
\hline PJ15_1202 & peptidase activity [GO:0008233] & 1.09716 \\
\hline PJ15_2954 & $\begin{array}{l}\text { serine-type endopeptidase activity } \\
\text { [GO:0004252] }\end{array}$ & 0.929888 \\
\hline PJ15_2971 & $\begin{array}{l}\text { ATP binding [GO:0005524]; } \\
\text { ATP-dependent peptidase activity } \\
\text { [GO:0004176]; sequence-specific DNA } \\
\text { binding [GO:0043565]; serine-type } \\
\text { endopeptidase activity [GO:0004252] }\end{array}$ & 0.883469 \\
\hline
\end{tabular}

AGG), isoleucine (AUA) and leucine (CUA) rare codons. The PJ15_1540 gene was successfully expressed, indicating that the "RIL" host strain was able to promote the recombinant protein production by overcoming the codon usage bias caused by the rare seventh (CUA coding leucine) and fifteenth (AUA) codons present in the gene sequence. To optimize the expression of the PJ15_1540 protein IPTG concentration, time and temperature of incubation were varied. The highest yield of the PJ15_1540 recombinant protein was obtained at $30^{\circ} \mathrm{C}$ and $2 \mathrm{mM}$ IPTG, after $4 \mathrm{~h}$ of incubation. The apparent molecular mass was consistent with the theoretical molecular mass of $44.15 \mathrm{kDa}$ calculated on the basis of the amino acid composition (Figure 3).

\section{Degrading Activity of the Recombinant PJ15_1540 Protein}

The activity of the recombinant PJ15_1540 protein was tested in cell lysate incubated overnight with $1 \mu \mathrm{g} / \mathrm{mL}$ of OTA. In this condition, PJ15_1540 protein was able to degrade 33\% of OTA 


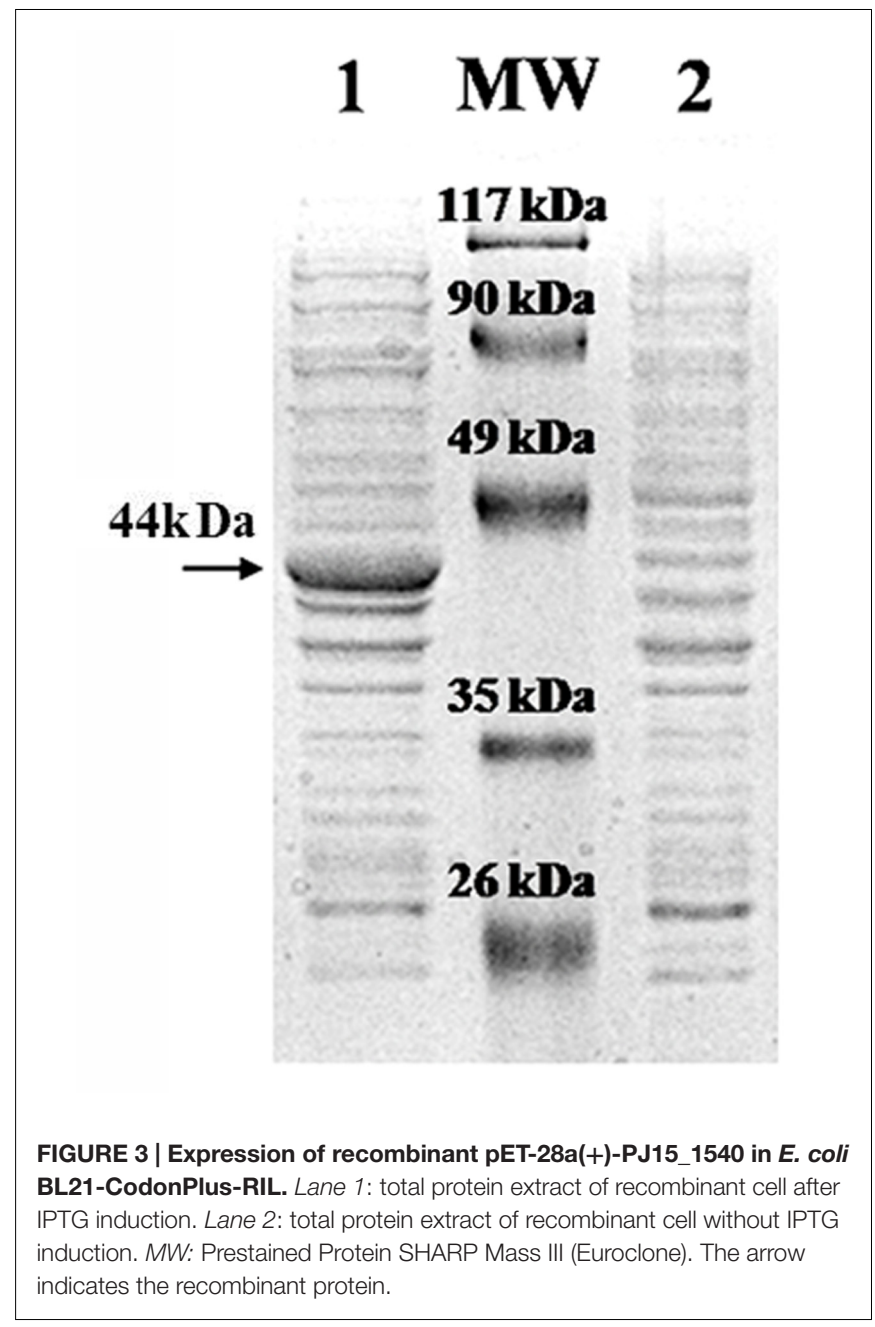

while a comparable production of OT $\alpha$ was shown (Figure 4). The supernatant liquid of induced cells did not show OTA degrading activity. This indicates that the recombinant protein is not secreted into the medium as confirmed by SDS-page (not shown).

\section{Pathway Analysis}

The enrichment analysis of the DE genes for Gene Ontology terms revealed the over-representation of a total of 59 pathways in 6 h-OTA-grown bacteria and 67 in 12 h-OTA-grown bacteria (Supplementary Data Sheets 3 and 4). The dysregulated pathways were grouped in eight functional categories on the basis of the Gene Ontology relations between terms; those pathways not clustering with others were included in a ninth category called other. The categories protein and amino acid metabolic process and transport, oxidoreductase activity, hydrolase activity, response to external stimulus and peptidase activity were dysregulated at $6 \mathrm{~h}$. The categories protein and amino acid metabolic process and transport, phenylalanine catabolism, oxidoreductase activity, transferase activity, hydrolase activity and organic acid metabolic process and transport were dysregulated at $12 \mathrm{~h}$ (Figure 5).

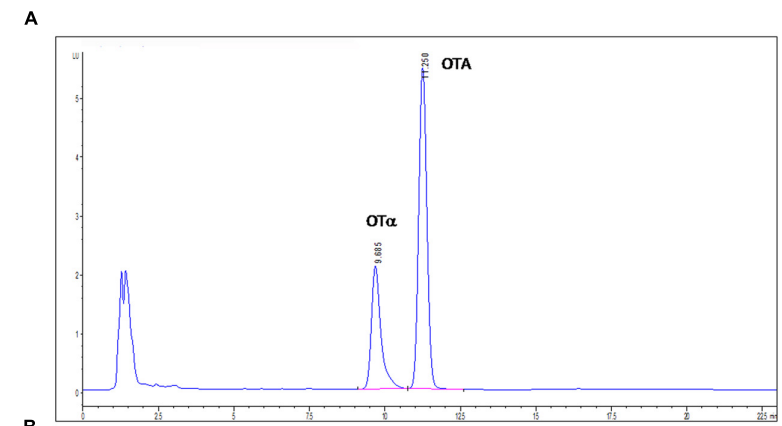

B

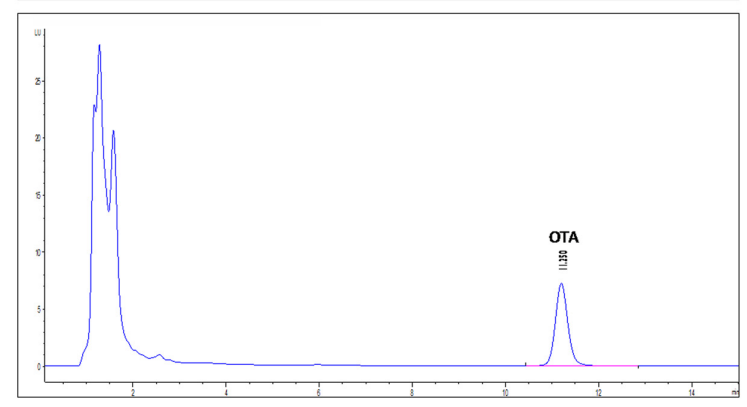

FIGURE 4 | High-performance liquid chromatography with fluorescence detection (HPLC-FLD) chromatograms of IPTG-induced (A) or not IPTG-induced (B) E. coli

BL21-CodonPlus-RIL-pET-28a(+)-PJ15_1540 cell lysates tested for OTA degradation. Retention times: OTA, $11.250 \mathrm{~min}$; OT $\alpha, 9.685 \mathrm{~min}$.

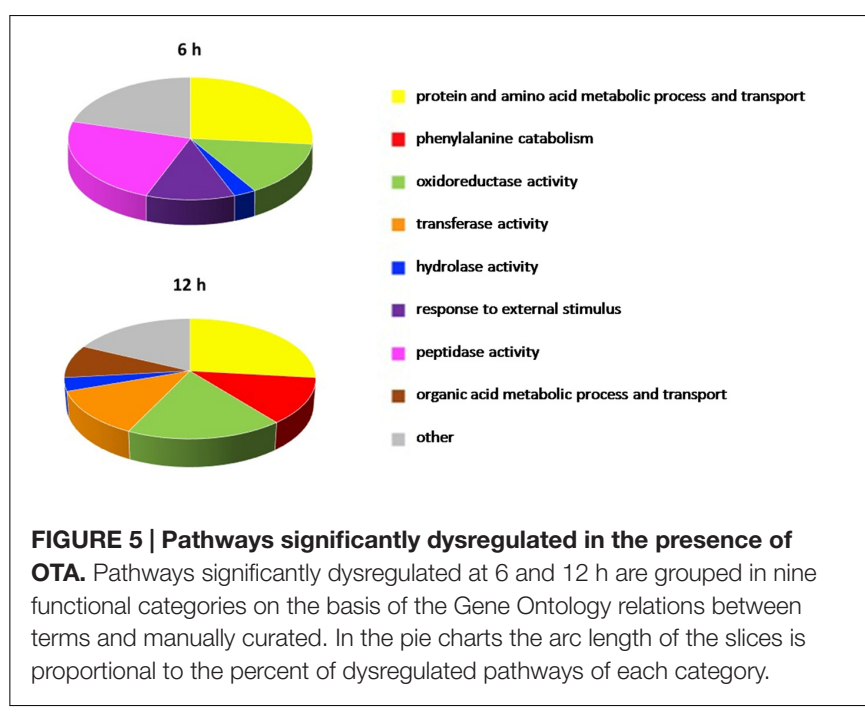

\section{DISCUSSION}

This work confirmed the ability of ITEM 17016 to degrade OTA into the non-toxic catabolic product OT $\alpha$. To investigate enzymes and pathways involved in the OTA degradation process, a comparative transcriptional analysis between bacteria grown in the presence or absence of OTA was performed.

The growth curve of ITEM 17016 was not influenced by the presence of OTA in the culture medium, suggesting that this mycotoxin does not exert any toxic effect on the cells, which 
is reasonable considering that OTA is present in their natural environment. Almost all the toxin content in the culture medium was converted to the hydrolyzed product OT $\alpha$. As already demonstrated by previous analysis (De Bellis et al., 2015; Fanelli et al., 2015), the microbial degradation through the hydrolysis of the amide bond results in the Phe molecule and OT $\alpha$. Our analyses did not reveal the presence of Phe in the medium, thus indicating that this molecule might be immediately used or further degraded by bacterial cells.

Among the up-regulated genes in the presence of OTA, six peptidase-coding genes were considered candidates for the OTA degradation reaction catalysis. PJ15_2703 codes for a metalloendopeptidase which catalyzes the hydrolysis of internal, alpha-peptide bonds in a polypeptide chain. In the reaction mechanism water acts as a nucleophile, one or two metal ions hold the water molecule in place, and charged amino acid side chains are ligands for the metal ions. PJ15_2971 codes for an ATP-dependent serine-type endopeptidase that mediates the selective degradation of mutant and abnormal proteins as well as certain short-lived regulatory proteins. PJ15_2954 codes for the putative serine-type endopeptidase sohB and PJ15_1202 codes for a putative protease. The strongest candidates were PJ15_1540 and PJ15_2908, coding for two serine-type D-AlaD-Ala carboxypeptidases showing $33 \%$ of sequence identity. Indeed, PJ15_1540 and PJ15_2908 proteins showed 29 and 25 identity, respectively, with ASAG1 carboxypeptidase of B. amyloliquefaciens, which was reported to degrade OTA in vitro (Chang et al., 2015).

The degrading activity of PJ15_1540 protein was confirmed by heterologous expression in E. coli. The recombinant protein was able to degrade OTA into OT $\alpha$, confirming the same mechanism of hydrolysis of the amide bond demonstrated for ITEM 17016.

In order to investigate at systemic level the OTA effect on the metabolism of the A. sp. neg1 strain, an enrichment analysis of the DE genes for Gene Ontology terms was completed. Consistent with the differentially up-regulated peptidases revealed under OTA presence, genes in the category peptidase activity were upregulated at $6 \mathrm{~h}$ of treatment but not at $12 \mathrm{~h}$, indicating an early activation of the mechanism leading to OTA hydrolysis.

After $6 \mathrm{~h}$ of incubation with OTA, activities and processes related to the category response to external stimulus were dysregulated with respect to the control. This suggested that, in this short period after OTA addition, the strain was sensing the changes in the environment as a stressing condition and activating responses to this stimulus which involves regulation of gene expression and protein activity (Starosta et al., 2014). However, this adaptation was completed within $12 \mathrm{~h}$, as demonstrated by the absence of this category among those which resulted altered after $12 \mathrm{~h}$.

Both at 6 and $12 \mathrm{~h}$ molecular functions and biological processes belonging to the protein and amino acid metabolic process and transport category were over-represented. In fact, Phe is one of the products of the degrading reaction and this category includes transmembrane transporters and specific amino acid transporters which are downstream the Phe catabolic pathway. Accordingly, among the gene sets displaying significant OTA-related dysregulation at $12 \mathrm{~h}$, seven pathways involved in Phe catabolism were highlighted. This suggests that Phe represents an energy source for ITEM 17016 and that the degrading reaction is followed by the modulation of further catabolic activities. In bacteria phenylalanine catabolism can proceed through different routes (Arias-Barrau et al., 2004; Teufel et al., 2010). Our results confirmed that there are several dysregulated pathways related to these catabolic processes, which are associated with the presence of OTA.

Pathway analysis also shows the alteration of 4hydroxyphenylpyruvate dioxygenase activity, which suggests that ITEM 17016 is capable of converting Phe into tyrosine by a pterin-dependent Phe hydroxylase. Then, tyrosine can be converted into 4-hydroxyphenylpyruvate, which is further transformed into 2,5-dihydroxyphenylacetate by a 4-hydroxyphenylpyruvate dioxygenase. By this way the aromatic ring is then split by a ring-cleaving dioxygenase, with the final production of fumarate and acetoacetate (Arias-Barrau et al., 2004; Teufel et al., 2010).

Phe could be alternatively metabolized via phenylacetate as extensively demonstrated for many bacterial species (Ferrández et al., 1998; Erb et al., 2008; Teufel et al., 2010). The downstream pathways of phenylacetate include the pathway of benzoate degradation (KEGG database ${ }^{5}$ ). We are led to assume that Phe metabolism in ITEM 17016 might also occur via this route, in accordance with the dysregulation of activities related to benzoate degradation due to the down-regulation of an hydroxybenzoate 3-monooxygenase.

The activity methylmalonate-semialdehyde dehydrogenase (acylating) related to propanoate catabolism, which is one of the pathways involved in degradation of aromatic compounds $\left(\right.$ KEGG database $^{5}$ ), was also altered after $12 \mathrm{~h}$ of treatment. In particular, PJ15_0600 gene coding for a methylmalonatesemialdehyde dehydrogenase resulted up-regulated with a $\log 2$-fold change of 6.8 , suggesting a strong involvement of this enzyme in the Phe catabolism. It should be noted that benzoate and propanoate are both organic acids, which is in accordance with the dysregulation of the organic acid metabolic process and transport category at $12 \mathrm{~h}$.

Moreover, we reported the over-representation of the oxidoreductase activity, which is consistent with the assumption that proteins involved in aerobic catabolism of aromatic compounds are often oxidoreductases (Parales and Resnick, 2006).

\section{CONCLUSION}

This paper describes a model of workflow for the development of a biotechnological application of enzymatic activities. A traditional microbiological approach combined with a comparative transcriptomic analysis has led to the identification of potential candidate genes and pathways involved in OTA degradation. Finally, the degrading activity of the carboxypeptidase PJ_1540 was validated in vitro in a heterologous system demonstrating its potential application in the food chain.

${ }^{5}$ http://www.genome.jp/kegg/ 


\section{ACCESSION NUMBERS}

The RNA sequencing raw data were submitted to NCBI under the accession number SRP078981.

\section{AUTHOR CONTRIBUTIONS}

All authors significantly contributed to this paper. VL, FF, and GM conceived and designed the experiments. VL and FF performed the growth experiment, degradation assays and RNA extraction. $\mathrm{MH}$ performed the chemical analysis. CM and CL performed the sequencing. VL and EP performed the differential expression and pathway analysis. GP and MiT supervised the bioinformatics workflow. MaT and FG performed the cloning and expression in heterologous system. All authors reviewed the paper. VL, FF, and AL were responsible for manuscript preparation and GM for the submission.

\section{ACKNOWLEDGMENTS}

The authors would like to thank Dr. Ramon Pacheco and Dr. Raquel Manzano for their informatics support during

\section{REFERENCES}

Abarca, M. L., Bragulat, M. R., Castella, G., and Cabanes, F. J. (1994). Ochratoxin A production by strains of Aspergillus niger var. niger. Appl. Environ. Microbiol. 60, 2650-2652.

Abrunhosa, L., Paterson, R. R., and Venâncio, A. (2010). Biodegradation of ochratoxin A for food and feed decontamination. Toxins 2, 1078-1099. doi: $10.3390 /$ toxins 2051078

Araguás, C., González-Penãs, E., and López de Cerain, A. (2005). Study on ochratoxin A in cereal-derived products from Spain. Food Chem. 92, 459-464. doi: 10.1016/j.foodchem.2004.08.012

Arias-Barrau, E., Olivera, E. R., Luengo, J. M., Fernández, C., Galán, B., García, J. L., et al. (2004). The homogentisate pathway: a central catabolic pathway envolved in the degradation of L-phenylalanine, L-tyrosine, and 3-hydroxyphenylacetate in Pseudomonas putida. J. Bacteriol. 186, 5062-5077.

Battilani, P., Pietri, A., and Logrieco, A. (2004). "Risk assessment and management in practice ochratoxin in grapes and wine," in Mycotoxins in Food Detection and Control, eds N. Magan and M. Olsen (Cambridge: Woodhead Publishing), 244-261.

Bayman, P., Baker, J. L., Doster, M. A., Michailides, T. J., and Mahoney, N. E. (2002). Ochratoxin production by the Aspergillus ochraceus group and Aspergillus alliaceus. Appl. Environ. Microbiol. 68, 2326-2329. doi: 10.1128/ AEM.68.5.2326-2329.2002

Breitholtz-Emanuelsson, A., Olsen, M., Oskarsson, A., Palminger, I., and Hult, K. (1993). Ochratoxin A in cow's milk and in human milk with corresponding human blood samples. J. AOAC Int. 76, 842-846.

Cappello, M. S., Stefani, D., Grieco, F., Logrieco, A., and Zapparoli, G. (2008). Genotyping by amplified fragment length polymorphism and malate metabolism performances of indigenous Oenococcus oeni strains isolated from Primitivo wine. Int. J. Food Microbiol. 127, 241-245. doi: 10.1016/j.ijfoodmicro. 2008.07.009

Chang, X., Wu, Z., Wu, S., Dai, Y., and Sun, C. (2015). Degradation of ochratoxin A by Bacillus amyloliquefaciens ASAG1. Food Addit. Contam. Part A Chem. Anal. Control Expo. Risk Assess. 32, 564-571. doi: 10.1080/19440049.2014. 991948

Covarelli, L., Beccari, G., Marini, A., and Tosi, L. (2012). A review on the occurrence and control of ochratoxigenic fungal species and ochratoxin A the transcriptomic analysis and Roberto Schena for his valuable chemical technical assistance. This work was financially supported by the Apulia Region Project cod. QCBRAJ6 "Biotecnologie degli alimenti per l'innovazione e la competitività delle principali filiere regionali: estensione della conservabilità e aspetti funzionali - BIOTECA." A fellowship for training abroad has been given to VL by Short Term Mobility (STM) 2015, National Research Council.

\section{SUPPLEMENTARY MATERIAL}

The Supplementary Material for this article can be found online at: http://journal.frontiersin.org/article/10.3389/fmicb. 2016.02162/full\#supplementary-material

DATA SHEET 1 | CuffDiff_6h. Output of CuffDiff comparative analysis between OTA-treated and control samples after $6 \mathrm{~h}$ of treatment.

DATA SHEET 2 | CuffDiff_12h. Output of CuffDiff comparative analysis between OTA-treated and control samples after $12 \mathrm{~h}$ of treatment.

DATA SHEET 3 | Pathway analysis_6h. Output of Bingo pathway enrichment analysis of the differentially expressed genes in 6h-OTA-grown bacteria.

DATA SHEET 4 | Pathway analysis_12h. Output of Bingo pathway enrichment analysis of the differentially expressed genes in 12h-OTA-grown bacteria.

in dehydrated grapes, non-fortified dessert wines and dried vine fruit in the Mediterranean area. Food Control 26, 347-356. doi: 10.1016/j.foodcont.2012. 01.044

De Bellis, P., Tristezza, M., Haidukowski, M., Fanelli, F., Sisto, A., Mulè, G. et al. (2015). Biodegradation of ochratoxin A by bacterial strains isolated from vineyard soils. Toxins 7, 5079-5093. doi: 10.3390/toxins7124864

Duarte, S., Pena, A., and Lino, C. (2010). A review on ochratoxin A occurrence and effect of processing of cereal and cereal derived food products. Food Microbiol. 27, 187-198. doi: 10.1016/j.fm.2009.11.016

Erb, T. J., Ismail, W., and Fuchs, G. (2008). Phenylacetate metabolism in thermophiles: characterization of phenylacetate-CoA ligase, the initial enzyme of the hybrid pathway in Thermus thermophilus. Curr. Microbiol. 57, 27-32. doi: $10.1007 / \mathrm{s} 00284-008-9147-3$

Fanelli, F., Chiara, M., Liuzzi, V. C., Haidukowski, M., Tristezza, M., Manzari, C., et al. (2015). Draft genome sequence of Acinetobacter sp. neg1 capable of degrading ochratoxin A. FEMS Microbiol. Lett. 362:fnv004. doi: 10.1093/femsle/ fnv004

Ferrández, A., Miñambres, B., García, B., Olivera, E. R., Luengo, J. M., and Garcı ìa, J. E. (1998). Catabolism of phenylacetic acid in Escherichia coli, characterization of a new aerobic hybrid pathway. J. Biol. Chem. 273, 25974-25986. doi: 10.1074/ jbc.273.40.25974

Frisvad, J. C., Thrane, U., Samson, R. A., and Pitt, J. I. (2006). Important mycotoxins and the fungi which produce them. Adv. Food Mycol. 571, 3-31. doi: 10.1007/0-387-28391-9_1

Gallo, A., Bruno, K. S., Solfrizzo, M., Perrone, G., Mulè, G., Visconti, A., et al. (2012). New insight into the ochratoxin A biosynthetic pathway through deletion of a nonribosomal peptide synthetase gene in Aspergillus carbonarius. Appl. Environ. Microbiol. 78, 8208-8218. doi: 10.1128/AEM. 02508-12

IARC (1993). "Some naturally occurring substances: food items and constituents, heterocyclic aromatic amines and mycotoxins," in IARC Monographs on the Evaluation of Carcinogenic Risks to Humans, Vol. 5 (Lyon: IARC Press), 489521.

Maere, S., Heymans, K., and Kuiper, M. (2005). BiNGO a Cytoscape plugin to assess overrepresentation of gene ontology categories in biological networks. Bioinformatics 21, 3448-3449. doi: 10.1093/bioinformatics/ bti551 
Makun, H. A., Gbodi, T. A., Akanya, H. O., Sakalo, A. E., and Ogbadu, H. G. (2007). Fungi and some mycotoxins contaminating rice (Oryza sativa) in Niger state Nigeria. Afr. J. Biotechnol. 6, 99-108.

Miraglia, M., and Brera, C. (2002). SCOOP-Report on Tasks for Scientific Cooperation, Assessment of Dietary Intake of Ochratoxin A By the Population of EU Member States. Available at http://europaeuint/comm/food/fs/scoop/327 enpdf

Parales, R. E., and Resnick, S. M. (2006). "Aromatic ring hydroxylating dioxygenases," in Pseudomonas: Molecular biology of emerging issues, Vol. 4, eds J. L. Ramos and R. C. Levesque (New York NY: Springer), 287-340.

Perši, N., Pleadin, J., Kovacevic, D., Scortichini, G., and Milone, S. (2014). Ochratoxin A in raw materials and cooked meat products made from OTAtreated pigs. Meat Sci. 96, 203-210. doi: 10.1016/j.meatsci.2013.07.005

Pfohl-Leszkowicz, A., and Manderville, R. A. (2007). Ochratoxin A: an overview on toxicity and carcinogenicity in animals and humans. Mol. Nutr. Food Res. 51, 61-99. doi: 10.1002/mnfr.200600137

Pitt, J. I. (1987). Penicillium viridicatum, Penicillium verrucosum and production of ochratoxin A. Appl. Environ. Microbiol. 53, 266-269.

Romani, S., Sacchetti, G., Chaves López, C., Pinnavaia, G. G., and Dalla Rosa, M. (2000). Screening on the occurrence of ochratoxin A in green coffee beans of different origins and types. J. Agric. Food Chem. 48, 3616-3619. doi: 10.1021/ jf990783b

Sambrook, J., Fritsch, E. F., and Maniatis, T. (1989). Molecular Cloning. New York, NY: Cold Spring Harbor Laboratory Press.

SCF (1998). Opinion of the Scientific Committee for food on OTA (Expressed on 17 September 1998). Available at: http://www.europe.eu.int/contam/food/fs/sc/scf/ out14_en.hml

Shannon, P., Markiel, A., Ozier, O., Baliga, N. S., Wang, J. T., Ramage, D., et al. (2003). Cytoscape: a software environment for integrated models of biomolecular interaction networks. Genome Res. 13, 2498-2504. doi: 10.1101/ gr.1239303

Somma, S., Perrone, P., and Logrieco, A. F. (2012). Diversity of black Aspergilli and mycotoxin risks in grape wine and dried vine fruits. Phytopathol. Mediterr. 51, 131-147.

Starosta, A. L., Lassak, J., Jung, K., and Wilson, D. N. (2014). The bacterial translation stress response. FEMS Microbiol. Rev. 38, 1172-1201. doi: 10.1111/ 1574-6976.12083
Stefanović, V., and Polenaković, M. (2009). Fifty years of research in Balkan endemic nephropathy: where are we now? Nephron Clin. Pract. 112, c51-c56. doi: $10.1159 / 000213081$

Taniwaki, M. H., Pitt, J. I., Teixeira, A. A., and Imanaka, B. T. (2003). The source of ochratoxin A in Brazilian coffee and its formation in relation to processing methods. Int. J. Food Microbiol. 82, 173-179. doi: 10.1016/S0168-1605(02) 00310-0

Teufel, R., Mascaraque, V., Ismail, W., Voss, M., Perera, J., Eisenreich, W., et al. (2010). Bacterial phenylalanine and phenylacetate catabolic pathway revealed. Proc. Natl. Acad. Sci. U.S.A. 107, 14390-14395. doi: 10.1073/pnas.1005399107

Trapnell, C., Williams, B. A., Pertea, G., Mortazavi, A., Kwan, G., van Baren, M. J., et al. (2010). Transcript assembly and quantification by RNA-Seq reveals unannotated transcripts and isoform switching during cell differentiation. Nat. Biotechnol. 28, 511-515. doi: 10.1038/nbt.1621

Varga, J., Kocsubé, S., Péteri, Z., Vágvölgyi, C., and Tóth, B. (2010). Chemical, physical and biological approaches to prevent ochratoxin induced toxicoses in humans and animals. Toxins 2, 1718-1750. doi: 10.3390/toxins2071718

$\mathrm{Wu}, \mathrm{T}$. D., and Nacu, S. (2010). Fast and SNP-tolerant detection of complex variants and splicing in short reads. Bioinformatics 26, 873-881. doi: 10.1093/ bioinformatics/btq057

Xiao, H., Madhyastha, S., Marquardt, R. R., Li, S., Vodela, J. K., Frohlich, A. A., et al. (1996). Toxicity of ochratoxin A, its opened lactone form and several of its analogs: structure-activity relationships. Toxicol. Appl. Pharmacol. 137, 182-192. doi: 10.1006/taap.1996.0071

Conflict of Interest Statement: The authors declare that the research was conducted in the absence of any commercial or financial relationships that could be construed as a potential conflict of interest.

Copyright @ 2017 Liuzzi, Fanelli, Tristezza, Haidukowski, Picardi, Manzari, Lionetti, Grieco, Logrieco, Thon, Pesole and Mulè. This is an open-access article distributed under the terms of the Creative Commons Attribution License (CC BY). The use, distribution or reproduction in other forums is permitted, provided the original author(s) or licensor are credited and that the original publication in this journal is cited, in accordance with accepted academic practice. No use, distribution or reproduction is permitted which does not comply with these terms. 